\title{
Effects of Ethanolic Extract of Hibiscus rosa-sinensis Leaves on Alloxan-Induced Diabetes with Dyslipidemia in Rats
}

\author{
Al Mamun, Saiful Islam, A.H.M. Khurshid Alam, Md. Aziz Abdur Rahman, Mamunur Rashid \\ Department of Pharmacy, University of Rajshahi, Rajshahi-6205, Bangladesh
}

\begin{abstract}
Diabetes associated dyslipidemia is one of the major causes of disability in western as well as in developing countries. The present study was designed to investigate the effect of ethanolic extract of Hibiscus rosa sinensis (EHBS) leaves on alloxan-induced diabetes with dyslipidemia in rats. Treatment of alloxan-induced diabetes with most effective observed dose $(2.0 \mathrm{mg} / \mathrm{kg}$ body weight $)$ of EHBS for 1 week significantly reduced glucose level, TC, TG and LDL-C, with the increase of HDL-C and weight of kidney, pancreas and liver when compared with diabetic rats. The observation was also made for consecutive 4 weeks to confirm the results obtained in one week model. Like one week, we got similar results when the treatment of alloxan-induced rats was done with the EHBS for 4 weeks. The results indicated that the EHBS leaves, in comparison with metformin, had profound hypoglycemic and hypolipidemic activities.
\end{abstract}

Key words: Antidiabetic, hypolipidemic, Hibiscus rosa-sinensis, Metformin.

\section{Introduction}

Diabetes mellitus is an endocrinal disorder with depleted insulin secretions, damaged pancreatic $\beta$-cells with altered carbohydrate, lipid and protein metabolism and additionally increased risk of complications of various vascular diseases. The aging populations, consumption of calorie-rich diets, obesity and sedentary lifestyles have led to a tremendous increase in the number of individuals with diabetes worldwide. The prevalence of diabetes for all age-groups worldwide was estimated $2.8 \%$ in 2000 and expected to be $4.4 \%$ in 2030 (Wild et al., 2004). In Bangladesh, about 5 million people are affected with diabetes (Habib et al., 2005). It is ranked seventh among the leading causes of death and third when all its fatal complications are taken into account. Hyperlipidemia associated atherosclerosis is the most common causes of death in diabetes (Trivedi et al., 2004).

Treatment of diabetes with commercially available drugs possesses some degree of adverse effects. However, antidiabetic drugs impose economical burden not only on the rural people but also on the modern society in Bangladesh (Ali et al., 2008). Therefore, the search for new, safe and easy to administer drugs remains a top priority. Drugs derived from medicinal plants have been brought into focus for meeting the goals of a wider coverage of health care delivery including diabetes with hyperlipidemia to all countries of the world. In Bangladesh, thousands of plant species are known to have medicinal values and the different parts of these medicinal plants have been used for different ailments since ancient times (Joshi et al., 2009). The importance of antidiabetic plants, in the development of economic and of effective treatment for diabetes, has been recognized by the World Health Organization (WHO Technical Report Series, 1985).

H. rosa-sinensis (Local name, Joba), is an evergreen shrub, belongs to the family Malvaceae. The flowers of $H$. rosa sinensis exhibited cardio-protective effect in rat (Gauthaman et al., 2006). Ethanolic extract of $H$. rosa sinensis (EHBS) leaves showed almost $100 \%$ post-coital antifertility activity (Vasudeva and Sharma, 2008). Moreover, EBHS flower aided wound healing in rat (Nayak et al., 2007). However, there is no published report about the effect of EHBS on diabetes associated hyperlipidemia. Therefore, we designed our present study to investigate the effect of EHBS leaves on alloxaninduced diabetes with hyperlipidemia in rats.

\section{Materials and Methods}

Collection of $H$. rosa-sinensis leaves: The fresh leaves of $H$. rosa-sinensis (Local name-Joba) were collected from Rajshahi University campus in August,

Correspondence to: Mamunur Rashid, Department of Pharmacy, University of Rajshahi, Rajshahi-6205, Bangladesh.

E-mail: mamun69jp@yahoo.com 
2009 and were botanically authenticated by a plant taxonomist from the Department of Botany, University of Rajshahi, Rajshahi, Bangladesh. A voucher specimen is deposited at the Department of Botany, University of Rajshahi, Bangladesh.

Preparation of crude extract: The crude ethanolic extract of H. rosa-sinensis leaves (EHBS) was prepared according to Alam et al. (2002). In brief, the leaves were cleaned and dried completely under mild sun and then ground into coarse powder with an electric grinder. The powdered material was then extracted with $96 \%$ ethanol at room temperature for 7 days with occasional shaking and stirring. The extract was filtered through a cotton plug and the filtrate was then concentrated with a rotary evaporator under reduced pressure to obtain crude extract.

Animal studies: All protocols for the animal experiments were reviewed and approved by the animal care and use committee of Institute of Biological Science, University of Rajshahi. Long-Evan's male rats weighing about 110-130 g, aged 2 months, were purchased from the Animal House of International Centre for Diarrhoeal Disease Research, Bangladesh (ICDDR,B). Prior to commencement of the experiments, all the rats were acclimatized to the new environmental condition for a period of one week. During the experimental period, the rats were kept in a well ventilated animal house at room temperature and were supplied with standard pellets from ICDDR,B and fresh drinking water. All the rats were kept in cages and maintained with natural 12 hour light and dark cycle. Alloxan was injected intra-peritonially (i.p.) in rats. Alloxan-induced diabetic rats were treated with i.p. injection of EHBS (0.5, 1 and $2 \mathrm{mg} / \mathrm{kg}$ bw) for dose selection. After completing the dose selection, alloxaninduced diabetic rats were treated with the most effective observed dose of EHBS ( $2 \mathrm{mg} / \mathrm{kg} \mathrm{bw}$ ) and metformin (150 mg/ $70 \mathrm{~kg} \mathrm{bw})$ for both 1 and 4 weeks.

Measurement of glucose level in blood: Experimental rats $(\mathrm{n}=6)$ were allowed to fast overnight, and rats were injected with alloxan $(120 \mathrm{mg} / \mathrm{kg}$ BW $)$ i.p. to induce diabetes. After one week of treatment with alloxan, rats with marked hyperglycaemia (fasting blood glucose $>13$ mmol) were selected for drug treatments. The EHBS (0.5, 1 and $2 \mathrm{mg} / \mathrm{kg} \mathrm{bw})$ and metformin $(150 \mathrm{mg} / 70 \mathrm{~kg}$, bw) were administered i.p. in diabetic rats. The blood samples were collected at $0,2,4$ and 6 hours, and blood glucose was determined using glucometer (OneTouch Horizone,
USA). The most effective observed dose of EHBS (2 $\mathrm{mg} / \mathrm{kg} \mathrm{bw})$ and metformin $(150 \mathrm{mg} / 70 \mathrm{~kg}$, bw) were administered i.p. daily for both 1 week and 4 weeks in alloxan-induced diabetic rats and blood glucose levels were determined using glucometer (OneTouch Horizone, USA).

Measurement of body weight, organ weight and lipid profiles: Treatment with EHBS and meformin for 1 and 4 week in diabetic rats were done. The changes in body weight, organ (liver, kidney and pancreas) weight, and serum lipid profile were determined. All assays were carried out in a Hitachi auto analyzer using diagnostic kits (GmbH, Germany) following standard procedures.

Statistical analysis: The results were expressed as mean \pm SEM. We used a one-way analysis of variance (ANOVA), followed by Dunnett's post-hoc test or students paired or unpaired $t$-test where appropriate. The statistical method applied in each analysis was described in each figure. Results were considered to be significant when $p$ values were less than $0.05(p<0.05)$.

\section{Results}

Effects of different doses of EHBS and metformin on alloxan-induced diabetic rat: To determine the effects of EHBS and metformin on alloxan-induced diabetic rats, we administered different doses of EHBS (0.5, 1 and $2 \mathrm{mg} / \mathrm{kg}$ BW) and metformin i.p. in diabetic rats. After administration of several doses of EHBS and metforminn, we observed that EHBS at different doses reduced the blood glucose level and the most significant reduction of blood glucose level was found from $17.5 \pm 0.69$ to $13.8 \pm$ $0.36 \mathrm{mmol}$ at $2 \mathrm{mg} / \mathrm{kg}$ bw among three doses, after 4 hours when compared with diabetic control rats. Metformin also decreased blood glucose level from $16.94 \pm 0.51$ to 12.90 $\pm 0.38 \mathrm{mmol}$, after 4 hours when compared with diabetic rats (Figure 1). We selected the dose at $2 \mathrm{mg} / \mathrm{kg}$ bw of EHBS for further study.

Effects of EHBS and metformin on glucose level, body \& organ weight and lipid profile in alloxan-induced diabetic rats for 1 and 4 week(s): During treatment of alloxan-induced diabetic rats with EHBS and metformin for 1 week, we observed that both EHBS and metformin significanty decreased blood glucose level. EHBS and metformin decreased blood glucose from $18.3 \pm 0.69$ to $13.25 \pm 0.53 \mathrm{mmol}$ and $18 \pm 0.51$ to $11.65 \pm 0.73 \mathrm{mmol}$, 
respectively when compared with diabetic control rats (Figure 2). In addition to reduce glucose level by treatment with EHBS and metformin in diabetic rats, both EHBS and metformin showed improvement in body weight, organ (liver, kidney and pancreas) weight when compared with diabetic control rats. The biochemical assays showed that serum total cholesterol (TC), triglyceride (TG) and LDL-cholesterol (LDL-C) levels were significantly increased with decreased level of serum HDL-cholesterol (HDL-C) in diabetic rats when compared to normal rats. After treatment with EHBS and metformin in diabetic rats, we found decreased levels of TC, TG and LDL-C with increased amount of HDL-C as compared to diabetic control rats (Table 1)

Table 1. Effects of 1 week treatment with EHBS and metformin on different physical and biochemical parameters of alloxaninduced diabetic rats.

\begin{tabular}{llcccc}
\hline $\begin{array}{c}\text { Serial } \\
\text { No. }\end{array}$ & Parameters & $\begin{array}{c}\text { Normal } \\
\text { control }\end{array}$ & $\begin{array}{c}\text { Diabetic } \\
\text { control }\end{array}$ & $\begin{array}{c}\text { Diabetic + EHBS } \\
(2.0 \mathrm{mg} / \mathrm{kg} / \mathrm{day})\end{array}$ & $\begin{array}{c}\text { Diabetic }+ \text { Metformin } \\
(150 \mathrm{mg} / \mathrm{kg} / \mathrm{day})\end{array}$ \\
\hline 1. & Body weight & $205.23 \pm 5.31$ & $165.15 \pm 6.1$ & $181.54 \pm 3.1^{*}$ & $172.38 \pm 1.39^{\dagger}$ \\
2. & Organ weight & & & & $5.23 \pm 0.25^{\dagger}$ \\
& Liver & $5.35 \pm 0.14$ & $4.05 \pm 0.29$ & $5.05 \pm 0.15^{*}$ & $0.58 \pm 0.02^{\dagger}$ \\
& Kidney & $0.61 \pm 0.02$ & $0.53 \pm 0 . .02$ & $0.65 \pm 0.02^{*}$ & $0.43 \pm 0.02^{\dagger}$ \\
3. & Pancreas & $0.41 \pm 0.06$ & $0.28 \pm 0.02$ & $0.41 \pm 0.03^{*}$ & $142.63 \pm 4.12^{\dagger}$ \\
& Lipid profile & & & $145.34 \pm 3.71^{*}$ & $151.45 \pm 3.38^{\dagger}$ \\
& TC & $137.51 \pm 6.28$ & $171.26 \pm 6.15$ & $135.53 \pm 2.38^{*}$ & $137.14 \pm 3.15^{\dagger}$ \\
& TG & $129.32 \pm 4.68$ & $183.72 \pm 5.12$ & $135.83 \pm 2.95^{*}$ & $57.54 \pm 0.98^{\dagger}$ \\
\hline
\end{tabular}

Data are presented as mean \pm SEM; $\mathrm{n}=6$ in each group, ${ }^{*} p<0.05$ and ${ }^{\dagger} p<0.05$ compared to diabetic control rats (ANOVA followed by Dunnett's test).

Table 2. Effects of 4 week treatment with EHBS and metformin on different physical and biochemical parameters of alloxaninduced diabetic rats.

\begin{tabular}{|c|c|c|c|c|c|}
\hline $\begin{array}{l}\text { Serial } \\
\text { No. }\end{array}$ & Parameters & $\begin{array}{l}\text { Normal } \\
\text { Control }\end{array}$ & $\begin{array}{l}\text { Diabetic } \\
\text { Control }\end{array}$ & $\begin{array}{l}\text { Diabetic + EHBS } \\
(200 \mathrm{mg} / \mathrm{kg} / \text { day })\end{array}$ & $\begin{array}{c}\text { Diabetic + Metformin } \\
(150 \mathrm{mg} / \mathrm{kg} / \text { day })\end{array}$ \\
\hline 1. & Body weight & $215.23 \pm 5.31$ & $155.15 \pm 6.1$ & $191.54 \pm 3.1^{*}$ & $186.38 \pm 1.39^{\dagger}$ \\
\hline \multirow[t]{4}{*}{2.} & Organ weight & & & & \\
\hline & Liver & $5.65 \pm 0.14$ & $4.25 \pm 0.29$ & $5.25 \pm 0.15^{*}$ & $5.43 \pm 0.25^{\dagger}$ \\
\hline & Kidney & $0.71 \pm 0.02$ & $0.43 \pm 0 . .02$ & $0.67 \pm 0.02 *$ & $0.68 \pm 0.02^{\dagger}$ \\
\hline & Pancreas & $0.61 \pm 0.06$ & $0.41 \pm 0.02$ & $0.85 \pm 0.03 *$ & $0.84 \pm 0.02^{\dagger}$ \\
\hline \multirow[t]{5}{*}{3.} & Lipid profile & & & & \\
\hline & $\mathrm{TC}$ & $149.71 \pm 6.15$ & $191.35 \pm 6.43$ & $153.65 \pm 3.92 *$ & $156.82 \pm 4.57^{\dagger}$ \\
\hline & TG & $131.91 \pm 4.54$ & $195.49 \pm 5.75$ & $133.55 \pm 2.61 *$ & $140.14 \pm 3.75^{\dagger}$ \\
\hline & LDL-C & $123.91 \pm 4.41$ & $180.38 \pm 4.77$ & $127.35 \pm 2.52 *$ & $137.14 \pm 3.15^{\dagger}$ \\
\hline & HDL-C & $65.21 \pm 0.57$ & $42.12 \pm 0.48$ & $62.86 \pm 0.39^{*}$ & $59.95 \pm 0.59^{\dagger}$ \\
\hline
\end{tabular}

Data are presented as mean \pm SEM; $n=6$ in each group, ${ }^{*} p<0.05$ and ${ }^{\dagger} p<0.05$ compared to diabetic control rats (ANOVA followed by Dunnett's test).

Like 1 week, induction of diabetes by alloxan for 4 weeks in rats significantly altered glucose level, body \& organ weight, and lipid profiles when compared with normal rats. To evaluate the effects of EHBS and metformin on glucose level, body and organ weight as well as lipid profile, we examined TC, TG, LDL-C and
HDL-C after i.p. injection of EHBS and metformin for 4 weeks in diabetic rats. After 4 weeks of treatment with EHBS and metformin, we found that both EHBS and metformin significantly decreased glucose level from $18 \pm$ 0.73 to $12.89 \pm 0.62 \mathrm{mmol}$ and $18 \pm 0.59$ to $11.56 \pm 0.43$ mmol, respectively when compared with normal rats 
(Figure 3). After daily administration of EHBS and metformin in rats for 4 weeks, we found improvement of body- and organ- (liver, kidney and pancreas) weight in diabetic treated rats when compared with diabetic rats. Moreover, treatment with EHBS and metformin in diabetic rats significantly decreased TC, TG and LDL-C levels with increased amount of HDL-C when compared with diabetic control rats (Table 2).

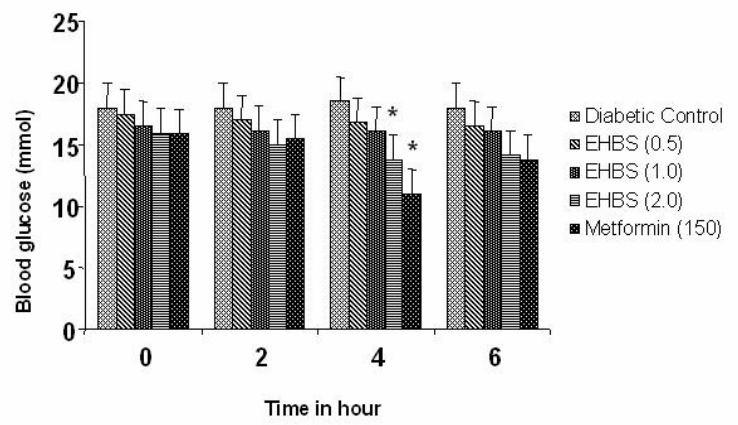

Figure 1. Effects of different doses of EHBS and metformin on blood glucose level in alloxan induced diabetic rats. All values were presented as mean \pm SEM; $n=6$ in each group, ${ }^{*} p<0.05$ compared to alloxan-induced diabetic rats (ANOVA followed by Dunnett's test). EHBS $=$ Ethanolic extract of $H$. rosa-sinensis

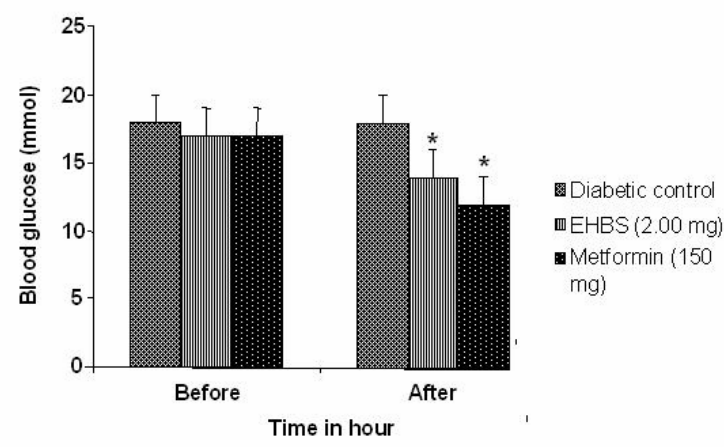

Figure 2. Effects of EHBS and metformin on glucose level in 1 week alloxan-induced diabetic rats. All values were presented as mean \pm $\mathrm{SEM} ; \mathrm{n}=4$ in each group, ${ }^{*} p<0.05$ compared to alloxan-induced diabetic rats (ANOVA followed by Dunnett's test). EHBS = Ethanolic extract of $H$. rosa-sinensis.

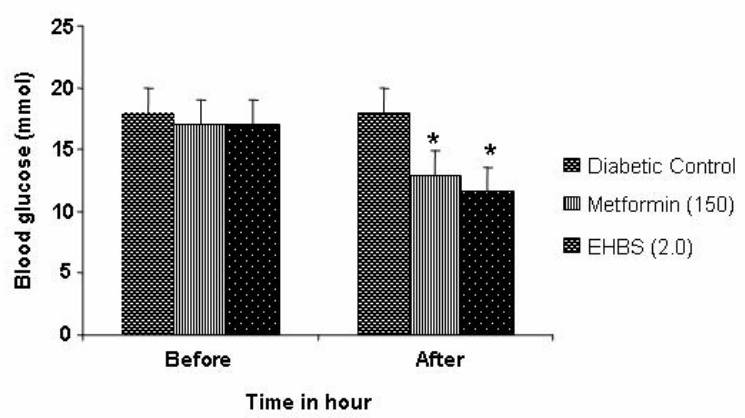

Figure 3. Effects of EHBS and metformin on glucose level in 4 weeks alloxan-induced diabetic rats. All values were presented as mean \pm SEM; $n=6$ in each group, ${ }^{*} p<0.05$ compared to alloxan-induced diabetic rats (ANOVA followed by Dunnett's test). EHBS $=$ Ethanolic extract of $H$. rosa-sinensis

\section{Discussion}

Diabetes associated dyslipidemia is the major cause of disability in western as well as in developing countries (Aqil et al., 2008). Prevalence of diabetes with their associated complications such as dyslipidemia is increasing, but the treatment alternatives are limited due to unwanted side effects and high costs (Dibianco, 2003). In the recent years, medicinal plants have come to view for the treatment of many diseases including diabetes owing to their easy availability and relatively minimal adverse effects. From our present investigation, EHBS is found to be effective medicinal plant in the treatment of diabetes with hyperlipidemia in rats. EHBS leaves significantly decreased blood glucose level in alloxan-induced diabetic rats compared with their respective control rats. Plant extract caused more significant hypoglycaemia in comparison with metformin. It has been reported that biguanide compounds produced hypoglycaemia in normal animals by reducing hepatic gluconeogenesis and by decreasing glucose absorption from gastrointestinal tract and also by increasing insulin sensitivity by increasing peripheral utilization of glucose. It is, therefore, conceivable that the mechanism of hypoglycaemic effect by EHBS might be similar to metformin.

Hypercholesterolemia and hypertriglyceridemia have been reported to occur in diabetic (Riyad et al., 1988; Tarfa et al., 1988; Sharma et al., 1996). In our study, TC and TG level increased significantly in diabetic control rats when compared with normal rats. In addition to TC and TG, LDL-C level was increased with decreased amount of HDL-C. Treatment of dyslipidemia with EHBS significantly reduced TC, TG and LDL-C and increased HDL-C level. Our results were consistent with the previously published reports (El-Dakhakhny et al., 2000). The hypolipidemic effect of EHBS was due to the synergistic action of its different constituents, including soluble fiber, sterols, flavonoids and high content of polyunsaturated fatty acids (Ali and Blunden, 2003). The exact mechanism of hypolipidemic action is not clear. However, it had been hypothesized that EHBS produced anti-hyperlipidemic effect by decreasing cholesterol synthesis, and more importantly by having antioxidant properties. Reduction in the body weight as well as organ weight in diabetic rats as well as in humans is well known. In case of diabetes, the body weight will increase when normal glycaemic levels is achieved which is particularly 
seen in sulfonyl ureas or insulin. EHBS increased body weight in diabetic animals, which might be due to increase insulin secretion and better glyceamic control.

In conclusion, EHBS showed hypoglycaemic and hypolipidemic activities in diabetic rats, which suggested that EHBS might be used as therapeutic alternative in the treatment of diabetes associated dyslipidemia.

\section{References}

Alam, A.H.M.K. et al. 2002. Chemical constituents of Hemisgraphis hirta T. Anders (Amaranthaceae). Pak. J. Biol. Sci. 5, 1264-1266.

Ali, B.H. and Blunden, G. 2003. Pharmacological and toxicological properties of Nigella sativa. Phytother. Res. 17, 299-305.

Ali, S.M., Fareed, A., Humail, S.M., Basit, A., Ahmedani, M.Y., Fawwad, A. and Miyan, Z. 2008. The personal cost of diabetic foot disease in the developing world-a study from Pakistan. Diabetes Med. 25, 1231-1233.

Aqil, S., Jaleel, A., Jaleel, F. and Basir, F. 2008. Comparison of adiponectin in ischemic heart disease versus ischemic stroke in diabetic patients. World Applied Sci. J. 3, 759- 762.

Dibianco, R. 2003. Update on therapy for heart failure. Am. J. Med. 115, 480-488.

El-Dakhakhny, M et al. 2000. Nigella sativa L. oil protects against induced hepatotoxicity and improves serum lipid profile in rats. Arzneimittel Forsch. 50, 832-836.

Gauthaman, K.K., Saleem, M.T.S., Thanislas, P.T., Prabhu, V.V., Krishnamoorthy, K.K., Devaraj, N.S., Somasundaram, J.S. 2006. Cardioprotective effect of the Hibiscus rosa sinensis flowers in an oxidative stress model of myocardial ischemic reperfusion injury in rat. $B M C$ Complementary and Alternative Med. 6, 32.
Habib, M.Y., Islam, M.S. and Khan, M.A. 2005. Herbal products: A novel approach for diabetic patients, Pak. $J$. Nut. 4, 17-21.

Joshi, B., Lekhak, S. and Sharma, A. 2009. Antibacterial property of different medicinal plants: Ocimum sanctum, Cinnamomum zeylanicum, Xanthoxylum armatum and Origanum majorana. Kathmandu University J. Sci. Eng. Tech. 5, 143-150.

Nayak, S.B., Raju, S.S., Orette, F.A. and Rao, A.V.C. 2007. Effects of Hibiscus rosa sinensis L (Malvaceae) on wound healing activity: A preclinical study in a sprague dawley rat. Int. J. Low Extrem. Wounds 6, 76-81.

Riyad, A., Abdul, G.A.S. and Suleiman, S.M. 1988. Effect of fenugreek and lupine seeds on the development of experimental diabetes in rats. Planta Medica 54, 286-290.

Sharma, S.R., Dwivedi, S.K. and Swarup, D. 1996. Hypoglycaemic and hypolipidaemic effects of Cinnamomum tamala Nees leaves. Indian J Exp Biol, 34, 372-374.

Tarfa. S.P., Joseph. P.K. and Augusti. K.T. 1988. Preliminary studies on the antidiabetic effects of cabbage (Brassica oleracea van capitata L.) oil on streptozotocin- diabetic rats. Curr. Sci. 57, 32-33.

Trivedi, N.A, Majumdar, B., Bhatt, J.D. and Hemavathy, K.G. 2004. Effects of shilajit on blood glucose and lipid profile in alloxan induced diabetic rats. Ind. J. Pharmacol. 36, 373386.

Vasudeva, N. and Sharma, S.K. 2008. Post-Coital antifertility activity of Hibiscus rosa-sinensis Linn. roots. Evid. Based Complement. Alternat. Med. 5, 91-94.

Wild, S., Gojka, R., Anders, G., Richard, S. and Hilary, K. 2004. Estimates for the year 2000 and projections for 2030 . Diabetes Care 27, 1047-1053.

World Health Organization. 1985. Diabetes mellitus: Report of a WHO Study Group. WHO Technical Report Series, p. 727. 